$12-2012$

\title{
Rotor Model Updating and Validation for an Active Magnetic Bearing Based High-Speed Machining Spindle
}

Adam C. Wroblewski

NASA Glenn Research Center

Jerzy T. Sawicki

Cleveland State University, j.sawicki@csuohio.edu

Alexander $\mathrm{H}$. Pesch

Cleveland State University

Follow this and additional works at: https://engagedscholarship.csuohio.edu/enme_facpub

Part of the Mechanical Engineering Commons

How does access to this work benefit you? Let us know!

Original Citation

Wroblewski, A.C., Sawicki, J.T. and Pesch, A.H., 2012, "Rotor Model Updating And Validation for an Active Magnetic Bearing Based High-Speed Machining Spindle," Journal of Engineering for Gas Turbines and Power- Transactions of the ASME, 134(12) pp. 122509.

This Article is brought to you for free and open access by the Mechanical Engineering Department at EngagedScholarship@CSU. It has been accepted for inclusion in Mechanical Engineering Faculty Publications by an authorized administrator of EngagedScholarship@CSU. For more information, please contact library.es@csuohio.edu. 


\title{
Adam C. Wroblewski \\ Jerzy T. Sawicki \\ Fellow ASME \\ Rotor Model Updating and Validation for an Active Magnetic Bearing Based High-Speed Machining Spindle
}

Alexander H. Pesch

Center for Rotating Machinery

Dynamics and Control (RoMaDyC),

Cleveland State University,

Cleveland, $\mathrm{OH} 44115-2214$

\begin{abstract}
This paper presents an experimentally driven model updating approach to address the dynamic inaccuracy of the nominal finite element (FE) rotor model of a machining spindle supported on active magnetic bearings. Modeling error is minimized through the application of a numerical optimization algorithm to adjust appropriately selected FE model parameters. Minimizing the error of both resonance and antiresonance frequencies simultaneously accounts for rotor natural frequencies as well as for their mode shapes. Antiresonance frequencies, which are shown to heavily influence the model's dynamic properties, are commonly disregarded in structural modeling. Evaluation of the updated rotor model is performed through comparison of transfer functions measured at the cutting tool plane, which are independent of the experimental transfer function data used in model updating procedures. Final model validation is carried out with successful implementation of robust controller, which substantiates the effectiveness of the model updating methodology for model correction.
\end{abstract}

\section{Introduction}

High-speed machining (HSM) has received considerable attention in recent years. It offers many attractive qualities such as shorter machining cycles, higher part accuracy, better surface finishes, and overall lower production costs in comparison to conventional machining techniques [1]. With proper application of high-speed machining, reduced cutting forces and increased material removal rates (MRR) are achieved [2].

Magnetically levitated spindles feature many benefits in comparison to spindles that operate on conventional rolling-element bearings [3]. Appropriate utilization of active magnetic bearings (AMBs) minimizes, or even completely eliminates, known problems and limitations associated with conventional rotor support types. The contactless levitation allows for significant increases in surface speeds and spindle diameters. Consequently, greater structural rigidity of the rotor is achievable. Advantages for AMB systems are numerous and are detailed in many texts, such as Ref. [4].

Active magnetic bearing rotor systems inherently require active feedback control to preserve levitation. Commonly, this duty is fulfilled by single-input single-output (SISO) controllers such as proportional-integral-derivative (PID), where each axis of control is controlled independently. However, enhancement in performance is achieved with more advanced multiple-input multiple-output (MIMO) robust controllers that operate by delivering crosscoupled control to all axes simultaneously. Effective implementation of robust control improves AMB performance; however, enhanced rotor modeling accuracy is required due to the modelbased nature of the robust controller design procedure.

Modeling of existing dynamic systems is often a nontrivial task and always has error associated with it. Manual correction of modeling error is a time consuming process that does not always promise improvement, especially for complicated structures. For this reason, an automated model updating routine is developed to improve model accuracy in terms of resonance frequencies as well as mode shapes, simultaneously. Model updating is as an

Contributed by International Gas Turbine Institute (IGTI) of ASME for publication in the Journal of Engineering for Gas Turbines and Power. Manuscript received June 21, 2012; final manuscript received June 30, 2012; published online October 25, 2012. Editor: Dilip R. Ballal. evolving field that has been pursued by many researchers [5] with various different approaches [6] and computational methods.

Dynamics of a structure are generally quantified by resonance frequencies and their corresponding mode shapes, which are measured with system identification methods. Natural frequencies are commonly extracted from frequency response functions (FRFs) through application of sine sweeps or impulse response measurements. Conversely, mode shapes are not easily measured since multiple discrete measurement points along the structure are required for each resonant frequency. Furthermore, mode shape measurements are not practical and in many cases are impossible due to accessibility limitations. The alternative to experimental mode shape measurement is utilization of antiresonance frequencies within typical FRFs [7]. This work presents the substantial improvement of model accuracy when model correction process utilizes antiresonance information.

To correct the FE rotor model's dynamic characteristics, carefully selected design variables are required. In the case presented here, the rotor's physical properties such as dimensions, mass, center of mass, and transverse and polar moments of inertia are known and were not altered. However, due to the complexity of the rotor components such as AMB rotors and the induction motor section, their local contributions to the rotor's global stiffness are less known. Consequently, only the modulus of elasticity for these structurally unknown finite elements was adjusted in the model updating routine.

The methodology in this work is outlined in the following tasks. First, open-loop spindle system identification is performed. Second, open-loop modeling and model updating methodology is developed. Following this section, a brief case study presents how excluding antiresonances in model updating affects the accuracy of the first two mode shapes. Finally, the validity of the updated model is demonstrated through the experimental implementation of a robust $\mathrm{AMB}$ controller.

\section{HSM Spindle and System Identification}

The platform for this study is a high-speed magnetically levitated machining spindle developed by Revolve Magnetic Bearings, a subsidiary of SKF, Inc., pictured in Fig. 1. It is supported by two radial and one thrust $\mathrm{AMB}$ and is designed to operate to a 


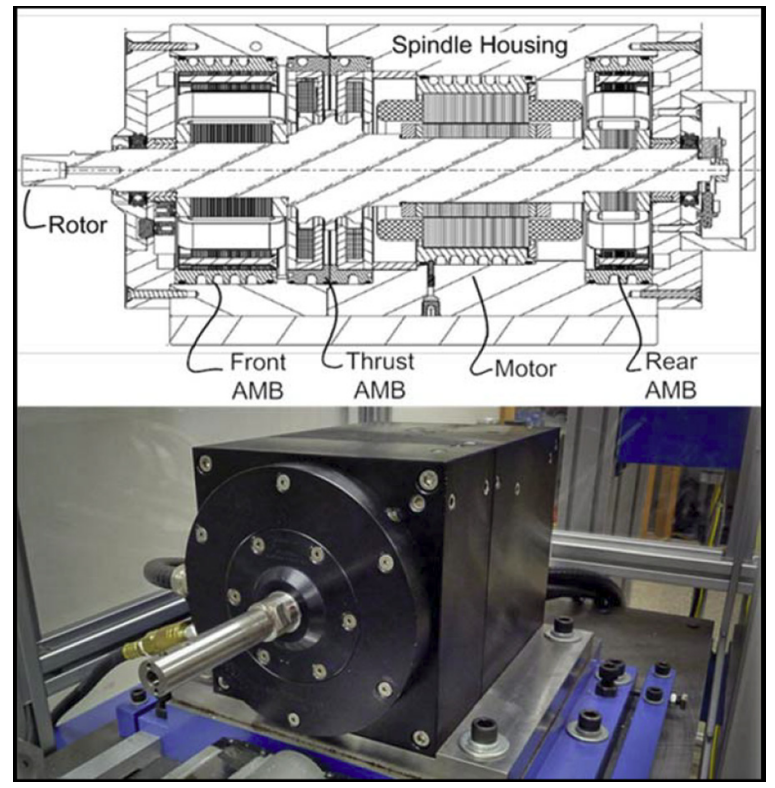

Fig. 1 Top: spindle cross section. Note that the drawing does not include the tool holder. Bottom: spindle photo.

maximum speed of $50,000 \mathrm{rpm}$. The maximum static loads that the front, rear, and thrust AMBs can support are $1400 \mathrm{~N}, 600 \mathrm{~N}$, and $500 \mathrm{~N}$, respectively. The $10 \mathrm{~kW}$ AC asynchronous induction motor rotor is located between the thrust AMB and the rear AMB. In the delevitated state, the rotor rests on backup ball bearings that provide a radial clearance of $254 \mu \mathrm{m}$.

Complete open-loop MIMO transfer functions (TFs) were acquired [8] using the existing AMB hardware to inject current sine sweeps during levitation under PID control over the range of $50 \mathrm{~Hz}$ to $4000 \mathrm{~Hz}$. The motivation for in situ testing is to circumvent the removal of the spindle rotor. Each radial AMB has two perpendicular control axes. The thrust axis is assumed to be uncoupled from the four radial axes, resulting in a four by four open-loop MIMO transfer function representing four radial axes of control. Equation (1) shows the relationship of $\mathbf{G}(s)$, where $\mathbf{G}(s)$ is a $4 \times 4$ matrix of transfer functions, and $\mathbf{u}$ (current) and $\mathbf{y}$ (displacement) are inputs and outputs, respectively [9].

$$
\mathbf{y}=\mathbf{G}(s) \mathbf{u}
$$

At each frequency of the sine sweep, four by four matrices of Fourier coefficients, $\mathbf{U}\left(\omega_{f}\right)$ and $\mathbf{Y}\left(\omega_{f}\right)$, are measured for each of the four current perturbation inputs and sensor displacement outputs. For each sine sweep perturbation, all inputs and outputs are recorded. Here, subscript $f$ represents discretized frequencies throughout the sine sweep signal input.

$$
\begin{aligned}
& \mathbf{U}\left(\omega_{f}\right)=\left[U_{i j}\left(\omega_{f}\right)\right]_{i, j=1 \ldots 4} \\
& \mathbf{Y}\left(\omega_{f}\right)=\left[Y_{i j}\left(\omega_{f}\right)\right]_{i, j=1 \ldots 4}
\end{aligned}
$$

\section{Tool Holder}

Connection

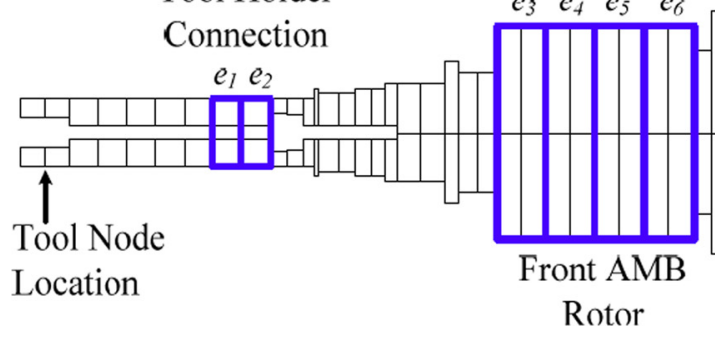

In this way, the signals are related as follows:

$$
\mathbf{Y}\left(\omega_{f}\right)=\mathbf{G}\left(j \omega_{f}\right) \mathbf{U}\left(\omega_{f}\right) \leftarrow
$$

To extract the open-loop transfer function, simple arithmetic is carried out to obtain

$$
\mathbf{G}\left(j \omega_{f}\right)=\mathbf{Y}\left(\omega_{f}\right) \mathbf{U}^{-1}\left(\omega_{f}\right) \leftarrow
$$

Although a total of sixteen TFs were acquired, only two were utilized for quantifying the rotor's dynamic behavior. The TFs with sine sweep signal injection and response measurement on the same axis tend to show the strongest resonance and antiresonance peaks. For this reason, the TFs with coaxial inputs/outputs (I/O) at the front $\left(\right.$ Bode $\left._{11}\right)$ and at the rear $\left(\right.$ Bode $\left._{33}\right)$ AMBs on a common axial plane were used throughout this work. They are presented in Fig. 4, as well as in the remaining TFs plots.

\section{Spindle Modeling and Model Updating}

The nominal FE spindle rotor model is a simplification of the real rotor. The rotor is difficult to accurately model due to its elaborate geometry and complicated structure. More specifically, modeling difficulties were found to be in the structural properties of the laminate materials in the AMB and motor rotors, the shrink fit interfaces, and the tapered attachment of the tool holder. Based on the known geometrical and mass information of the spindle rotor, a finite element rotor model was developed as illustrated in Fig. 2. The finite element rotordynamic code used in this work did not account for the unknown interactions of shrink fit interfaces, multiple material layers, inhomogeneous materials, small geometrical details, and so on, which additively contribute to modeling inaccuracy.

A modally reduced state-space representation was most convenient for control oriented application. Modal truncation was applied in order to retain the first three flexible modes because the system identification measurements were not capable of capturing higher frequencies. Five modes $m_{t}$ were retained, which included two rigid-body modes $m_{r}$ and the first three flexible modes $m_{f}$. In state-space representation, ordinary differential equations are represented in matrix format for programming convenience. They are also considered to be more numerically stable than models represented by transfer function equations. The state-space matrices for the free-free (ff) rotor, $\mathbf{A}_{f f}, \mathbf{B}_{f f}$, and $\mathbf{C}_{f f}$ are generated and are utilized for subsequent mathematical model manipulations [10]. The open-loop model is illustrated in Fig. 3 with solid lines.

Initial open-loop modeling applied nominal material parameters to all elements in the FE model. However, due to the unknown stiffness contribution of the several rotor components, the nominal model was shown to be not representative of the real spindle rotor. Figure 4 illustrates the significant error of the nominal spindle model when compared with system identification data. Comparing modeled and experimental data should be performed on as many domains as possible, such as spatial, frequency, and time response $[11,12]$. Several techniques have been adopted to quantify the comparison and are categorized into either visual or numerical

Fig. 2 The FE rotor model with subregions designating the structurally uncertain elements 


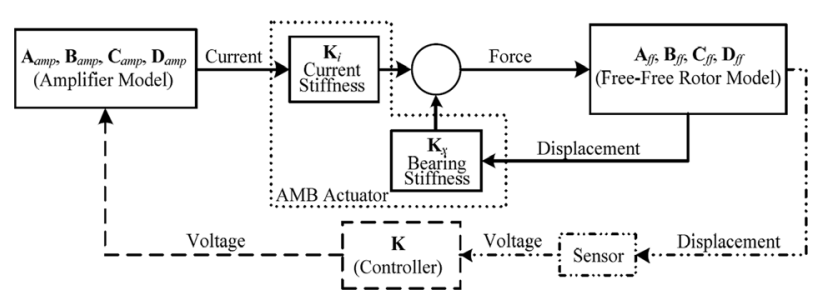

Fig. 3 The open-loop model (solid lines)
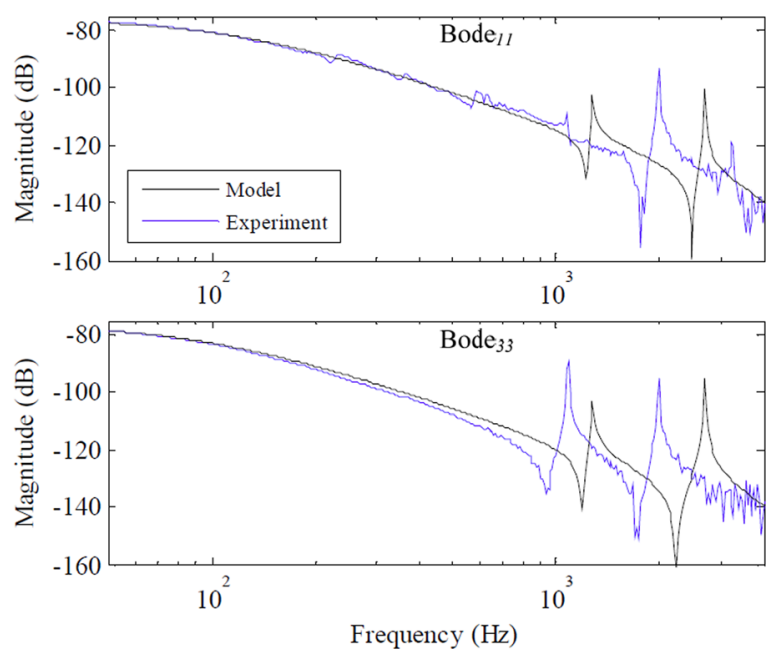

Fig. 4 Transfer functions from the front (top) and rear (bottom) AMBs illustrating the nominal model and experimental data

assessments [13]. Here, quantification of modeling error is based on differences of the corresponding resonance and antiresonance frequencies between modeled and experimental data.

Model updating was employed to correct for the modeling simplifications through adjustments of the modulus of elasticity of the fifteen FE model regions seen in Fig. 2. Since computational effort must be taken into account in order for the model updating routine to finish in timely manner, a balance between solution flexibility and the number of design variables was necessary. For this case, fifteen design variables (corresponding to fifteen finite elements) provided a reasonable balance. The only bound imposed on the design variables was that they must be positive real values.

Initialization of the updating routine began with the definition of the design variables $e_{p}$, represented by the vector, e, in Eq. (5), where subscript $p$ denotes the individual design variable and $n$ is the total number of design variables. All other finite elements have fixed nominal properties and were assumed to be correct. Note that the vector e in Eq. (5) was initially populated with nominal modulus of elasticity values for the specific material.

$$
\mathbf{e} \in \mathbb{R}^{n} \text { so that }\left\{e_{p}\right\}_{p=1 \ldots n}
$$

The vector $\mathbf{e}$ is utilized to calculate the model's resonance and antiresonance peaks for subsequent comparison with experimental data. The variables $r_{i j, k}$ and $a_{i j, k}$ in Eq. (6) represent the corresponding resonance and antiresonance errors, respectively.

$$
\begin{aligned}
& r_{i j, k}=\left|\hat{\omega}_{i j, k} \quad \tilde{\omega}(\mathbf{e})_{i j, k}\right| \\
& a_{i j, k}=\downarrow|\downarrow| \hat{\omega}_{i j, k} \quad{ }^{a} \tilde{\omega}(\mathbf{e})_{i j, k} \mid
\end{aligned}
$$

The subscripts $i$ and $j$ reference the individual transfer function in Fig. 4 and the subscript $k$ denotes the corresponding flexural resonance or antiresonance peak number. The variables $\hat{\omega}_{i j, k}, \tilde{\omega}(\mathbf{e})_{i j, k} \in \mathbb{R}$ are the experimental and calculated values for resonances, respectively. The variables ${ }^{a} \hat{\omega}_{i j, k},{ }^{a} \tilde{\omega}(\mathbf{e})_{i j, k} \in \mathbb{R}$ are the experimental and calculated values for antiresonances, respectively, denoted with the superscript $a$. The frequency values for $\hat{\omega}_{i j, k}$ and ${ }^{a} \hat{\omega}_{i j, k}$ are extracted manually from the experimentally acquired transfer function $\mathbf{G}_{0, i j}$ and are hard coded into expressions $r_{i j, k}$ and $a_{i j, k}$ since they are fixed and do not change for this system. The frequency values $\tilde{\omega}(\mathbf{e})_{i j, k}$ and ${ }^{a} \tilde{\omega}(\mathbf{e})_{i j, k}$ are extracted based on calculation of poles and zeros of the open-loop plant model $\mathbf{P}_{0}(\mathbf{e})_{i j}$. The error, in the most general form, between $\mathbf{G}_{0}$ and $\mathbf{P}_{0}(\mathbf{e})$ is calculated by Eq. (7). The terms $b_{x}$ and $b_{y}$ represent which of the 16 experimental MIMO Bode plots are utilized. The exponents $w_{k, r}$ and $w_{k, a}$ are the weighting factors for each error value, which can be different based on the mode error priority decided by the engineer.

$$
\operatorname{err}\left(r_{i j, k}, a_{i j, k}\right)=\sum_{i=1}^{b_{x}} \sum_{j=1}^{b_{y}} \sum_{k=1}^{m_{f}}\left[r_{i j, k}^{w_{k, r}}+a_{i j, k}^{w_{k, a}}\right](
$$

The open-loop plant, experimental data, and error function are augmented into a black box operator $B(\mathbf{e})$. The operator $B(\mathbf{e})$ is referred to as a black box function because the optimization routine [14] disregards the inner operations of the several tasks within. Operator $B(\mathbf{e})$ requires an input vector e generated by the optimization routine that then simply outputs an error value err. The smaller the error value, the more accurate the solution is. The tasks within $B(\mathbf{e})$ begin with the calculation of the current model's poles and zeros based on the most recently adjusted e vector, then calculation of the pole and zero error between the calculated and experimental data, and finally, evaluation of the error function $\operatorname{err}\left(r_{i j, k}, a_{i j, k}\right) \in \mathbb{R}$, which outputs an error value. The error value is directed to the optimization routine that generates another candidate e vector for the next loop evaluation. The error minimization loop continues until stopping criteria have been met, which can be a subjective matter since a perfect model may never be achieved.

The Nelder-Mead nonlinear unconstrained optimization method [15] is employed to adjust the e vector to minimize the error function. This is a well-established, derivative-free optimization algorithm that can handle discontinuous, nonsmooth functions. This method minimizes the real design variables using only the objective function's error value. The search for a minimizer proceeds through recursive updates of the simplex vertices. Each simplex operation depends on the objective function evaluation, where the simplex is updated through a progression of reflection, expansion, contraction, and shrinkage operations. It is known as a relatively robust and simple routine, which is why it was initially applied to this problem, noting that a global minimum may not be achieved. The generalized minimization problem is defined to find the vector e such that

$$
\min _{\mathbf{e} \in \mathbb{R}^{n}} B(\mathbf{e}) \leftarrow
$$

On average, approximately 400-500 iterations were necessary to generate an adequate solution. The final solution's transfer functions are plotted in Fig. 5, showing nearly zero error in resonance and antiresonance peaks. Note that the third antiresonance peak in Bode $_{33}$ is not identifiable and was not included in the error function.

Since the updated model utilizes antiresonance data in the objective function, mode shapes and resonance frequencies have been simultaneously corrected. Figure 6 plots the good agreement of the updated model's mode shapes with the available experimental mode shape data. Notice that mode shape measurements were only possible at the exposed rotor length as well as AMB sensor locations. The experimental mode shape measurements were acquired by injecting sine wave perturbation at resonance frequency into an $\mathrm{AMB}$ during levitation and translating an 

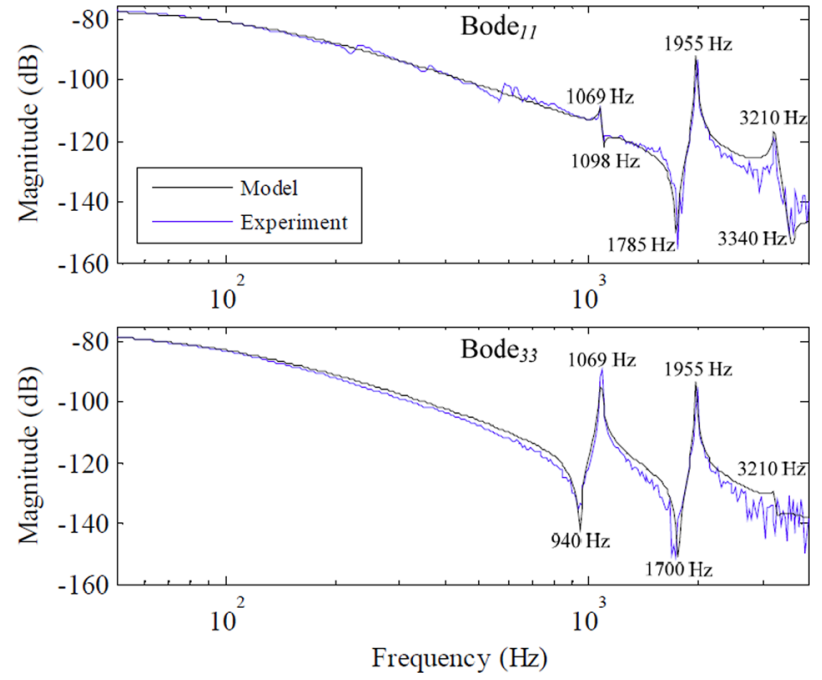

Fig. 5 Transfer functions from the front (top) and rear (bottom) AMBs illustrating the updated model and experimental data

external sensor along the exposed rotor length to make discrete point measurements.

It is important to note that this model updating problem's objective function included three resonance frequency errors and a total of five antiresonance frequency errors. Resonance frequencies are the same for all measurement locations (AMBs), unlike antiresonances, which are unique from one transfer function measurement to the next. Although two TFs were utilized in this problem, more objectives could be applied in the form of antiresonance errors from cross-coupled axis, such as Bode ${ }_{13}$, where the input signal injection is at the front AMB and the response measurement is at the rear AMB. The mode shape accuracy of the updated model would improve but with the increased computational cost due to increased difficulty of minimizing additional errors. Conversely, fewer errors may be used such as by utilizing only information in Bode $_{11}$, however, mode shape accuracy will suffer. In this work, utilization of coaxial TFs from the front and rear AMBs was found to be adequate.

It should be pointed out that all operations were performed in the levitated, nonrotating state. This inherently neglects effects of rotation such as gyroscopic effects and potential centrifugal expansion of shrink-fit components, which may induce speeddependent dynamic changes. At this stage of the research, it is assumed that speed-dependent changes do not significantly alter spindle performance at high speed.

\section{Antiresonances in Model Updating}

Antiresonance frequencies play an important role in defining the rotor's flexible behavior across a range of frequencies. Due to the burdensome nature of measuring the complete mode shape of

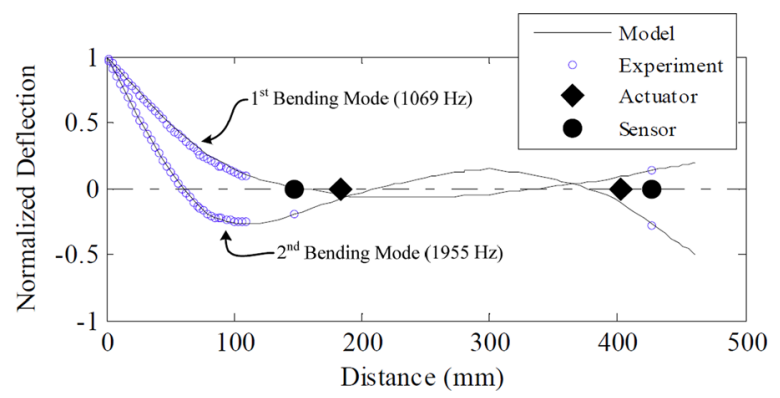

Fig. 6 Mode shapes of the first two bending modes illustrating the updated model and the experimental data
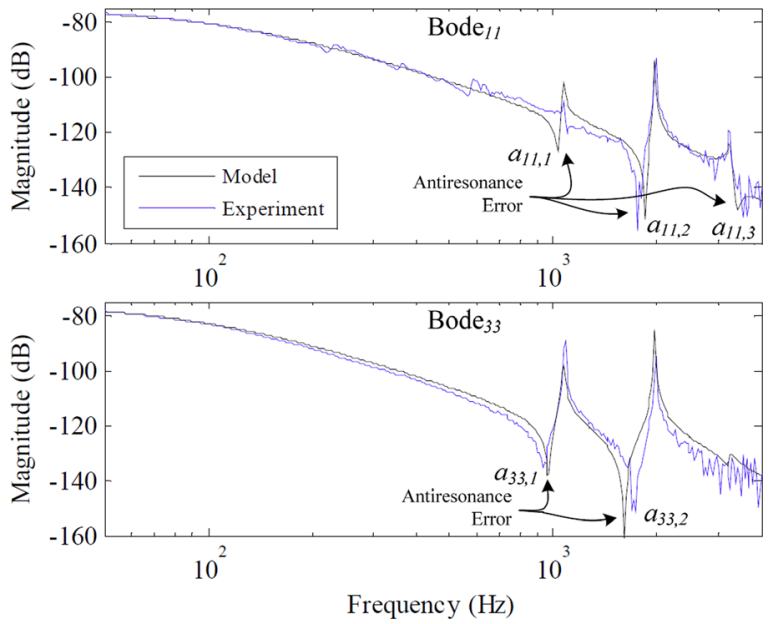

Fig. 7 Transfer functions from the front (top) and rear (bottom) AMBs illustrating the updated model (excluding antiresonances) and experimental data

the spindle rotor for several resonance frequencies, utilization of antiresonance frequencies is a practical alternative. They are easily obtained from the system identification transfer functions using existing AMB hardware. The utilization of antiresonance frequencies (in place of explicit mode shape measurements) is motivated by the anticipated routine changes of the tool holder and tool for each machining process. With each tool change, the spindle's controlled plant is changed and, thus, requiring a new corresponding robust controller. Since this may be a routine operation, spindle disassembly and reassembly for traditional testing is impractical, thus necessitating an in situ methodology.

Rather than representing complete mode shapes, antiresonances represent frequencies at which bending nodes are located at the corresponding AMB sensor or actuator [7]. This information provides the engineer with knowledge of several discrete locations and frequencies where vibrations are effectively zero. Note that antiresonances are sensitive to locations of inputs and outputs in a system. An assumption behind the utilization of antiresonances for model updating is that the rotor structure performs linearly through the frequency range of interest. In other words, since the rotor's flexural dynamics is defined at several (nonresonant) frequencies, it is assumed that the rotor will continue to behave linearly at resonant frequencies and produce accurate mode shapes.

The following section demonstrates the case where the model updating routine only corrects the resonance frequencies and disregards all antiresonance frequencies. Figure 7 plots the updated model against the same experimental TF data as presented in Fig. 4.

Table 1 lists the labeled errors of the antiresonances from Bode $_{11}$ and Bode 33 plotted in Fig. 7. Note that $a_{33,3}$ is not identifiable and was not considered.

Figure 8 illustrates mode shapes corresponding to the inaccurately updated model represented by the TFs plotted in Fig. 7. Although the resonance frequencies exhibit nearly zero error, the mode shapes for the first two resonance frequencies show

Table 1 Error Values of five antiresonance frequencies from Bode $_{11}$ and Bode ${ }_{33}$

\begin{tabular}{lr}
\hline \hline Antiresonance error & $\mathrm{Hz} / \%$ \\
\hline$a_{11,1}$ & $61 / 5.5$ \\
$a_{11,2}$ & $76 / 4.3$ \\
$a_{11,3}$ & $120 / 3.5$ \\
$a_{33,1}$ & $40 / 4.2$ \\
$a_{33,2}$ & $115 / 6.7$ \\
\hline \hline
\end{tabular}




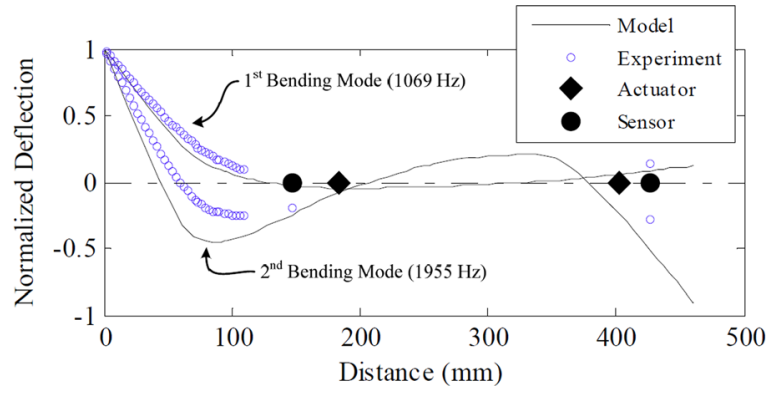

Fig. 8 Mode shapes of the first two bending modes for the updated model (excluding antiresonances) and the experimental data

significant disparity in comparison to the same experimental mode shape measurements presented in Fig. 6.

\section{Updated Model Validation}

Validity of the updated open-loop model was demonstrated through successful implementation of a MIMO $\mu$-synthesized controller [9] that was generated utilizing the updated rotor model represented by Fig. 5. Since the $\mu$-controller is generated based on the spindle model, robust levitation of the real machining spindle is achieved only when the model is of high fidelity and representative of the real test platform.

The $\mu$-controller used in this work was generated with performance weighting functions and uncertainty specifications shown in Table 2. This $\mu$-controller is referred to as the benchmark $\mu$-controller in which subsequent controllers in the study are compared against. The design intent behind this controller was to sustain robust levitation under a variety of excitation sources such as unbalance forces due to rotation over the entire speed range ( 0 to $50,000 \mathrm{rpm}$ ) and impulse/step inputs at the tool location. The performance weighting functions and uncertainty specifications were assumed to be reasonable for benchmarking purposes. Real machining operations were not considered in this work.

The tool location dynamic stiffness measurements were performed on the nonrotating spindle rotor in the levitated state utilizing the $\mu$-controller, which are plotted in Fig. 9 alongside the corresponding model simulations. The tool location dynamic stiffness of the spindle levitated with the PID controller is included as well for comparison. The measurements were conducted at the tool plane using an instrumented hammer and capacitance probe. The input and output responses of the instrumented hammer and capacitance probe were recorded by a spectrum analyzer that was used to calculate the dynamic stiffness, which is the inverse of the compliance.

Several observations are made from Fig. 9. First, the overall tool location stiffness magnitude for the $\mu$-controller case is notably higher over the low frequency range as well as at the spindle's maximum speed $(833 \mathrm{~Hz})$ in comparison to the PID controller case. Note that because Fig. 9 is the inverse of the dynamic compliance plot, the spindle's resonances are annotated as the minimum peaks, which illustrate the rotor's maximum compliance.

Table 2 Specifications of the benchmark $\mu$-synthesis controller

\begin{tabular}{lrcr}
\hline \hline & Tool & Front AMB & Rear AMB \\
\hline Max DC load $(\mathrm{N})$ & 40 & 100 & 100 \\
Max HF load $(\mathrm{N})$ & 5 & 10 & 10 \\
Max DC displacement $(\mu \mathrm{m})$ & 50 & 10 & 10 \\
Max HF displacement $(\mu \mathrm{m})$ & 10 & 30 & 30 \\
First mode uncertainty $(\%)$ & 1 & & \\
Second mode uncertainty $(\%)$ & 1 & & \\
\hline
\end{tabular}

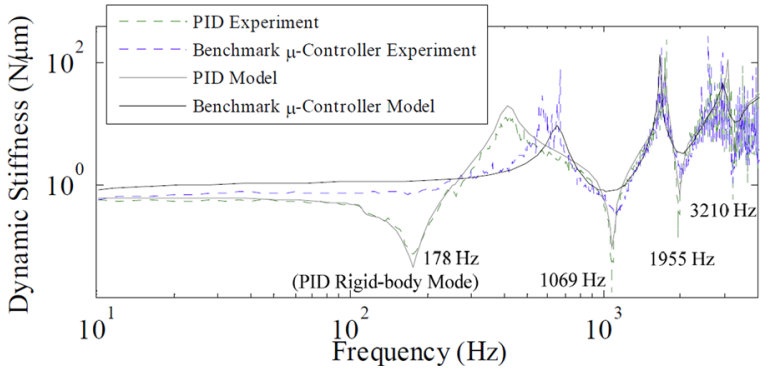

Fig. 9 Experimental and simulated stiffness of the spindle at the tool plane for the $\mu$-controller and the PID controller

Second, the responses of the three flexible modes, $1069 \mathrm{~Hz}$, $1955 \mathrm{~Hz}$, and $3210 \mathrm{~Hz}$, are substantially damped by the $\mu$-controller, when compared with the PID controller. The suppression of resonance frequency responses can be a substantial performance and safety improvement in machines that have to pass through critical speeds. Since the maximum running speed $(833 \mathrm{~Hz})$ is well below the first rotor resonance frequency, passing though critical speeds is not a concern for this spindle. Third, rigid-body body modes are not identifiable when levitated with the $\mu$-controller. Similar to the suppression of rotor resonance frequency response, the absence of rigid-body modes is a significant performance and safety enhancement.

\section{Conclusions}

The model updating routine requires a carefully chosen objective function to solve for a plant model that is representative of experimental data. Typically, models are corrected in such a manner that corrects for resonance frequencies; however, it was demonstrated to be not completely sufficient in terms of mode shape accuracy. Since mode shapes are burdensome to measure, antiresonance frequencies are an effective alternative, particularly in cases of routine alterations to the controlled plant such as with a machining spindle. For the purpose of model updating, these terms within the objective function provide the simultaneous correction of resonances as well as the corresponding mode shapes.

To ensure that the updated plant model is representative of the real system, model validation operations were required. Final updated model validations were performed through implementation of a $\mu$-controller generated based on the updated FE rotor model in conjunction with electrical models of the surrounding components. The concept for this was that since the $\mu$-controller was designed based on the updated plant model provided, the rotor will not levitate robustly if the plant model was not adequate for the $\mu$-synthesis [16]. The successful experimental implementation of the $\mu$-controller demonstrated the high fidelity of the updated open-loop plant model. Higher values of tool tip dynamic stiffness, in the presence of rigid body modes and flexible modes, were achieved with $\mu$-synthesis control. The automated open-loop model updating routine presents itself as a practical and an effective technique for model correction for subsequent use in robust controller generation. Further, as presented in Ref. [7], the updated plant model provides accurate time domain response predictions at the tool plane.

\section{Nomenclature}

$$
\begin{aligned}
a & =\text { ssuperscript representing antiresonance } \\
a_{i j, k} & =\text { tantiresonance error } \\
\mathbf{A}, \mathbf{B}, \mathbf{C} & =\text { state-space representation } \\
\mathrm{AC} & =\text { talternating current } \\
\mathrm{AMB} & =\text { tactive magnetic bearing } \\
B(\mathbf{e}) & =\text { black-box operator } \\
\mathrm{DC} & =\text { tow frequency }
\end{aligned}
$$


$e_{p}=$ tesign variables (modulus of elasticity)

err $=$ terror value

$\mathbf{e}=$ wector of design variables, modulus of elasticity

$f f=$ stubscripts representing free-free

$\mathrm{FE}=$ finite element

$\mathbf{G}=$ \&gyroscopic matrix

$\mathbf{G}_{0}=$ experimental open-loop transfer function

$\mathbf{G}(s)=$ matrix of experimental transfer functions

$\mathbf{G}\left(j \omega_{f}\right)=$ matrix of experimental open-loop transfer functions

$\mathrm{HF}=$ high frequency

HSM $=$ high-speed machining

$i, j=$ stubscripts representing input and output axes

$\mathrm{I} / \mathrm{O}=$ input/output

$k=$ stubscript for flexible resonance or antiresonance peak

$m_{f}=$ sumber of flexible modes

$m_{t}=$ total number of modes

$m_{r}=$ trumber of rigid-body modes

MIMO $=$ multiple-input multiple-output

$\mathrm{MRR}=$ material removal rate

$n=$ mumber of design variables

$p=$ subscript representing design variable number

$\mathbf{P}_{0}=$ smodeled open-loop transfer function

$\mathrm{PID}=$ proportional-integral-derivative

$r_{i j, k}=$ resonance error

SISO $=$ single-input single-output

$\mathrm{TF}=$ transfer function

$\mathbf{u}=$ wector of input signals

$\mathbf{U}\left(\omega_{f}\right)=$ Fourier coefficients of signal inputs

$\mathrm{y}=$ wector of output displacement

$\mathbf{Y}\left(\omega_{f}\right)=$ Fourier coefficients of response outputs

$w_{k, r}=$ weighting factor for resonance error

$w_{k, a}=$ wweighting factor for antiresonance error

$\omega_{f}=$ frequency

$\hat{\omega}_{i j, k}=$ experimental resonance frequency

$\tilde{\omega}_{i j, k}=$ kealculated resonance frequency

${ }^{a} \hat{\omega}_{i j, k}=$ texperimental antiresonance frequency

${ }^{a} \tilde{\omega}_{i j, k}=$ ecalculated antiresonance frequency

\section{References}

[1] Koelsch J. R., 2001, "High Speed Machining; A Strategic Weapon," Machine Shop Guide, Smith Publishing Inc., Garden City, NY.

[2] Badrawy S., 2001, "Cutting Dynamics of High Speed Machining," Wolf Trecks, 8(1), pp. 24-26.

[3] Chen, M., and Knospe C. R., 2007, "Control Approaches to the Suppression of Machining Chatter Using Active Magnetic Bearings," IEEE T. Contr. Sys. T., 15(2), pp. 220-232.

[4] Schweitzer, G., and Maslen E. H., 2009, Magnetic Bearings: Theory, Design, and Application to Rotating Machinery, Springer-Verlag, Berlin/Heidelberg.

[5] Friswell, M., and Mottershead J. E., 1995, Finite Element Model Updating in Structural Dynamics, Kluwer Academic Publishers, Dordrecht, The Netherlands.

[6] Xiong, Y., Chen, W., Tsui K.-L., and Apley D. W., 2008, "A Better Understanding of Model Updating Strategies in Validating Engineering Models," 49th AIAA/ASME/ASCE/AHS/ASC Structures, Structural Dynamics, and Materials Conference, Schaumburg, IL, April 7-10, Paper No. AIAA 2008-2155.

[7] Wroblewski A. C., 2011, "Model Identification, Updating and Validation of an Active Magnetic Bearing High-Speed Machining Spindle for Precision Machining Operation," Doctoral dissertation, Cleveland State University, Department of Mechanical Engineering, Cleveland, OH.

[8] Sawicki, J. T., and Maslen E. H., 2008, "Accurate Identification of Plant Model for Robust Control of an AMB Machine Tool Spindle," Ninth International Conference on Motion and Vibration Control (MOVIC 2008), Munich, Germany, September 15-18.

[9] Sawicki, J. T., and Maslen E. H., 2007, "Rotordynamic Response and Identification of AMB Machining Spindle," ASME Turbo Expo Conference, Montreal, Canada, May 14-17, ASME Paper No. GT2007-28018.

[10] Sawicki, J.T., and Maslen, E.H., Bischof, K.R., 2007, "Modeling and Performance Evaluation of Machining Spindle With Active Magnetic Bearings," J. Mech. Sci. Tech., 21(6), pp. 847-850.

[11] Marwala T., 2010, Finite Element Model Updating Using Computational Intelligence Techniques: Applications to Structural Dynamics, Springer-Verlag, London.

[12] Ewins D. J., 2001, Modal Testing: Theory, Practice and Application, Research Studies Press Ltd., Hertfordshire, England.

[13] Wu Y.-X., 1999, "Sensitivity-Based Finite Element Model Updating Methods With Application to Electronic Equipments," Ph.D. thesis, Polytechnique de Mons, Mons, Belgium.

[14] Yang W. Y., Cao W., Chung T.-S., and Morris J., 2005, Applied Numerical Methods Using MATLAB, John Wiley \& Sons, Inc., Hoboken, NJ.

[15] Nelder, J. A., and Mead R., 1965, "A Simplex Method for Function Minimization," Comput. J., 7(4), pp. 308-313.

[16] Sawicki, J. T., and Maslen E. H., 2008, "Toward Automated AMB Controller Tuning: Progress in Identification and Synthesis," 11th International Symposium on Magnetic Bearings (ISMB-11), Nara, Japan, August 26-29. 